\title{
Integrating laboratory and epidemiological techniques for population-based surveillance of HIV strains and drug resistance in Canada
}

\author{
Gayatri C Jayaraman PhD MPH ${ }^{1}$, Chris P Archibald MDCM FRCPC ${ }^{1}$, Lee Lior MD MSc ${ }^{2}$, \\ Donald Sutherland MD MCommH MSc(Epi ${ }^{1}$
}

$\mathrm{H}$ IV is among the most genetically variable of human pathogens. Two major factors contribute to this genetic diversity: the error-prone activity of reverse transcriptase, which is estimated to introduce an average of one error/genome/replication cycle (1), and recombination, which occurs at a rate of about $2 \% /$ kilobase/replication cycle (2).

With the advent of international collaborations using powerful new tools that allow for the analyses of nucleotide sequence information, it became apparent that the initial classification of HIV into HIV-1 and HIV-2 based on geographic distribution was inadequate. We now recognize that HIV-1 can be divided into three major phylogenetic groups: 'M' (major), 'O' (outlier) and more recently, 'N' (new). The vast majority of isolates cluster in the $M$ group. Based on sequencing the envelope gene, env, 10 phylogenetic subtypes (A to J) have been identified within this group, with subtypes A to E (also referred to as the circulating recombinant $\mathrm{A} / \mathrm{E}$ ) being the most common (3). The general pattern of subtype distribution by geographic location is shown in Table 1.

The second major group of HIV-1, group 0 , is found mainly in Cameroon and Gabon, and differs from the $\mathrm{M}$ group by as many as $50 \%$ of residues (4). The N group of HIV-1 was isolated in Cameroon, with genetic characteristics of both the simian immunodeficiency virus and HIV-1 (M group) (5).

Although there has been no systematic surveillance for ge- netic diversity of HIV strains in Canada, existing studies on high risk populations suggest that HIV-1 subtype B is the most common subtype found in this country. Bernier et al (6) have conducted analyses on HIV-1 sequence diversity among 17 infected injection drug users (IDUs) and among five men who have sex with men (MSM) residing in Montreal, and all sequences were of HIV-1 subtype B. As a part of an outbreak study in Newfoundland, Montpetit et al (7) analyzed serological samples from $31 \mathrm{HIV}$-positive persons of both sexes, comprising approximately $25 \%$ of known HIV-positive persons in the province, to determine the extent of HIV-1 subtype variation (7). All samples tested were of HIV-1 subtype B. Strain analysis has been carried out on samples from 13 MSM, two IDUs and two heterosexuals, recruited through POLARIS in Ontario (8). All have been found to be subtype B. The British Columbia Centre for Excellence in HIV/AIDS in Vancouver, British Columbia, has conducted genetic analysis of HIV linked to VIDUS (9). All 64 IDUs tested were infected with subtype B.

Despite the predominance of HIV-1 subtype B, non-B subtypes have also been reported in Canada. As early as 1995, HIV-1 subtype A was identified in an African-born male, who moved to Canada in 1983 (10). Studies by the British Columbia Centre for Excellence in HIV/AIDS suggest that non-B subtypes represent at least $4 \%$ of HIV infections among

\footnotetext{
${ }^{1}$ Bureau of HIVIAIDS, STD and TB; ${ }^{2}$ Bureau of Global Surveillance and Field Epidemiology, Laboratory Centre for Disease Control, Health Canada, Ottawa, Ontario

Correspondence: Dr G Jayaraman, Division of HIV Epidemiology, Bureau of HIV/AIDS, STD and TB, Laboratory Centre for Disease Control, Health Canada, Tunney's Pasture, AL O900B1, Ottawa, Ontario K1A OL2. Telephone 613-954-0881, fax 613-954-5414,

e-mail gayatri jayaraman@hc-sc.gc.ca
} 
individuals starting therapy (11). HIV-1 subtypes A, C, and D have been identified. Non-B subtypes were associated with poor outcomes upon initiation of treatment (12).

\section{THE CANADIAN HIV STRAIN AND DRUG RESISTANCE SURVEILLANCE PROGRAM}

The Canadian HIV Strain and Drug Resistance Surveillance Program (CHSDRSP), based in the Bureau of HIV/AIDS, STD and TB, Laboratory Centre for Disease Control, Health Canada, Ottawa, Ontario, was initiated to characterize and monitor the genetic diversity of the HIV epidemic in Canada. CHSDRSP consists of two principle components: laboratorybased genetic information and epidemiological information. It is designed to serve as an integrated mechanism for the analysis of HIV genetic characteristics as they relate to the epidemiology of HIV, addressing the concerns of public health authorities, primary care physicians and researchers. The four goals of CHSDRSP are as follows.

\section{To assess circulating strains}

Strain variation in Canada and around the world needs to be identified and well understood to develop effective vaccines, to understand the transmissibility of strains and to understand the pathogenicity of the strains. Because vaccines may be effective against certain subtypes only, it is important to know the distribution of the viral subtypes in Canada to determine the effectiveness of proposed vaccines and to target future vaccine development and testing.

While there is no clear evidence of a correlation between HIV-1 subtype and the biological behaviour of the virus, some studies suggest the different subtypes of HIV may have different transmission patterns. For example, in Thailand, although two different HIV-1 subtypes, E and B, were introduced at approximately the same time, the proportion of subtype $E$ has increased in most population groups (13). This and other observations showing changes in the proportion and distribution of subtypes over time suggest that certain non-B subtypes, including subtype $\mathrm{E}$, may be transmitted more effectively during heterosexual intercourse (14). If confirmed, this would be cause for concern because the introduction of subtype $\mathrm{E}$ to Canada could significantly change the nature of the HIV epidemic in this country.

Preliminary work by Alexander et al (15) indicates that B and non-B HIV subtypes respond similarly to antiretroviral treatment. The effects of viral subtype on response to therapy and the public health implications are also of special interest.

\section{To assess genetic markers for drug resistance}

Monitoring and assessing the patterns of primary drug resistance, such as is done for resistant forms of other sexually transmitted diseases including gonorrhea, can enhance the utility of CHSDRSP. Determining the extent of resistant viral genotypes among those recently diagnosed with HIV infection would be useful in developing treatment strategies and new interventions to prevent the spread of HIV-1 among the Canadian population.
TABLE 1

Geographic distribution of group ' $M$ ' subtypes of HIV-1

\begin{tabular}{ll}
\hline HIV-1 (M) subtype & Geographic area \\
\hline A, D & Central and East Africa \\
B & Americas, Europe, India, Thailand \\
C & India, South and Central Africa \\
E & Central African Republic, India, Thailand \\
F & Brazil, Congo, Eastern Europe \\
G & Gabon, Taiwan, Congo \\
H & Congo, Gabon \\
I & Cyprus \\
J & Congo \\
\hline
\end{tabular}

To assess patterns of transmission

Given the impact of international travel and migration on the spread of HIV, it is likely that non-B subtypes will continue to be introduced into Canada. If unusual strains are detected, this could contribute to the knowledge of infections acquired outside of the country. Genetic linkages could be done in a community or remote area to determine what proportion of HIV is brought in from outside the area. Combining strain surveillance data with enhanced information gathering from individuals could also be very useful in investigating transmission patterns. Additionally, for special studies outside the strict parameters of surveillance, genetic sequencing could establish the probability that two or more individuals share a common virus.

\section{To enhance the safety of the blood supply}

To enhance the safety of the blood supply, HIV screening tests need to detect reliably the circulating strains in this country. The precedent for this goal was the discovery of HIV-2 and highly divergent group $O$ strains of HIV-1 which required modification of some serological screening tests by adding these new antigens to ensure detection. CHSDRSP will monitor the circulating strains of HIV- 1 to facilitate the discovery of new HIV variants that might not be detected by current HIV screening tests. Based on the knowledge of circulating HIV types, and vigorous laboratory testing of individuals with unusual clinical presentations and of samples showing unusual serological, polymerase chain reaction (PCR) or viral culture results, modifications can be made in current tests to ensure that all HIV-1 positive persons in Canada are detected upon testing. There are many obvious implications of reliably screening and detecting new HIV strains, including protecting the blood supply, ensuring accurate diagnosis and understanding HIV transmission patterns.

\section{CHSDRSP INITIATIVES \\ Laboratory References Services, Bureau of HIV/AIDS, STD and TB, LCDC}

Provincial health laboratories (PHLS) and Canadian Blood Service are involved in HIV testing of individuals, and as such are key partners to CHSDRSP. A number of factors that result in unusual serological responses are relatively well established (seroconversion and cross-reactivity to HIV-2) and are 
TABLE 2

HIV-1 subtype distribution of nine "difficult to diagnose" samples submitted to the National Laboratory for HIV Genetics for viral subtyping

\begin{tabular}{lcc}
\hline Province & Subtype & $\begin{array}{c}\text { Year sample } \\
\text { submitted to NLHG }\end{array}$ \\
\hline Alberta & $\mathrm{B}$ & 1998 \\
Manitoba & $\mathrm{C}$ & 1998 \\
& $\mathrm{C}$ & 1999 \\
Ontario & $\mathrm{B}$ & 1999 \\
& $\mathrm{~A}$ & 1998 \\
Nova Scotia & $\mathrm{B}$ & 1999 \\
Newfoundland & $\mathrm{A}$ & 1999 \\
\hline
\end{tabular}

NLHG National Laboratory for HIV Genetics

recognized by the testing laboratories. Other conditions that are less well understood can also cause unusual serological test results. These samples may show cross-reactivity or represent divergent strains, as seen in 1993 with the failure of some diagnostic kits in France to detect HIV-1 subtype O (16).

The National Laboratory for HIV Genetics (NLHG) and National Laboratory for HIV References Services (NLHRS) serve PHLs by testing and identifying samples showing unusual serological, PCR or viral culture testing results. This ongoing partnership serves as an additional surveillance arm of CHSDRSP and is crucial for monitoring divergent HIV strains in Canada.

In the past two years, five provinces have submitted serological samples to the NLHG from individuals that were difficult to diagnose. These samples were analyzed using the techniques mentioned below, and the subtypes were determined. The results of these analyses are shown in Table 2 . The majority of the samples submitted were of subtypes A and $\mathrm{C}$. Of note, two subtype A samples and one subtype $C$ sample were obtained from individuals born or infected in Africa.

\section{CHSDRSP Pilot Study}

At a national consensus meeting in January 1998 to discuss the feasibility of the goals of CHSDRSP, working groups including federal, provincial and territorial stakeholders recommended piloting the surveillance methodologies at one or more sites before implementing CHSDRSP nationwide. The five sites included in this pilot phase were British Columbia,
Alberta, Saskatchewan, Manitoba and Newfoundland. This section describes interim results from subtype analysis of archived samples taken for HIV diagnosis or viral load testing in the CHSDRSP pilot sites.

Methodology - Laboratory: Archived sera collected from newly diagnosed HIV infected persons or plasma collected from persons coming for viral load testing were sent by the PHLs at the pilot sites to the NLHG at LCDC for subtype analysis.

The protocol for HIV-1 subtyping was as follows. Viral particles in $200 \mu \mathrm{L}$ of serum were pelleted at $21,000 \times g$ for $1 \mathrm{~h}$ at $4^{\circ} \mathrm{C}$, the supernatant aspirated and the RNA from the viral pellet isolated using the NucliSens silica extraction system (Organon Teknika, Toronto, Ontario) derived from Boom et al (17). Single tube reverse transcription PCR (RT-PCR) were performed using env specific primers ED3 (HXB2 position 59565985) and ED14 (HXB2 position 7960-7931) with the Calypso RT-PCR kit (Bio/Can, Mississauga, Ontario). DNA from cells and dried blood spots were amplified by PCR also using these env specific primers. Secondary, nested PCR reactions, using $5 \mu \mathrm{L}$ of primary PCR product, were performed with primers ED5 (HXB2 position 6556-6581) and ED12 (HXB2 position 7822-7792) in QIAgen Master Mix (Mississauga, Ontario). Simultaneous bidirectional sequencing reactions were prepared using the ThermoSequenase kit (Amersham, Montreal, Quebec) and IRD-labelled primers ES7 (HXB2 position 70017020), and ES8 (HXB2 position 7667-7647) yielding a complete double stranded sequence for 650 bases of the envelope gene (V2-V5 region). The reactions were run on a LI-COR $4200 \mathrm{~L}$, and the results were assembled and analyzed using AlignIR (Li-Cor, Lincoln, Nebraska), Sequencher (Gene Codes, Ann Arbor, Michigan) and software developed by NLHG.

Epidemiology: In addition to the laboratory specimen, corresponding epidemiological information was also collected and sent to the Division of HIV Epidemiology, Bureau of HIV/AIDS, STD \& TB. The data included information on age, sex, ethnicity, year of positive test, city where test was conducted, type of serological specimen collected and risk factors associated with HIV infection. However, because the majority of data was extracted retrospectively from medical records, not all the information mentioned above was available for each sample. Subtype information for each sample from the NLHG was linked to the corresponding epidemiological information using the unique specimen identifiers, and further analysis was con-

TABLE 3

Distribution of HIV-1 subtypes from the Canadian HIV Strain and Drug Resistance Surveillance Program pilot study by province

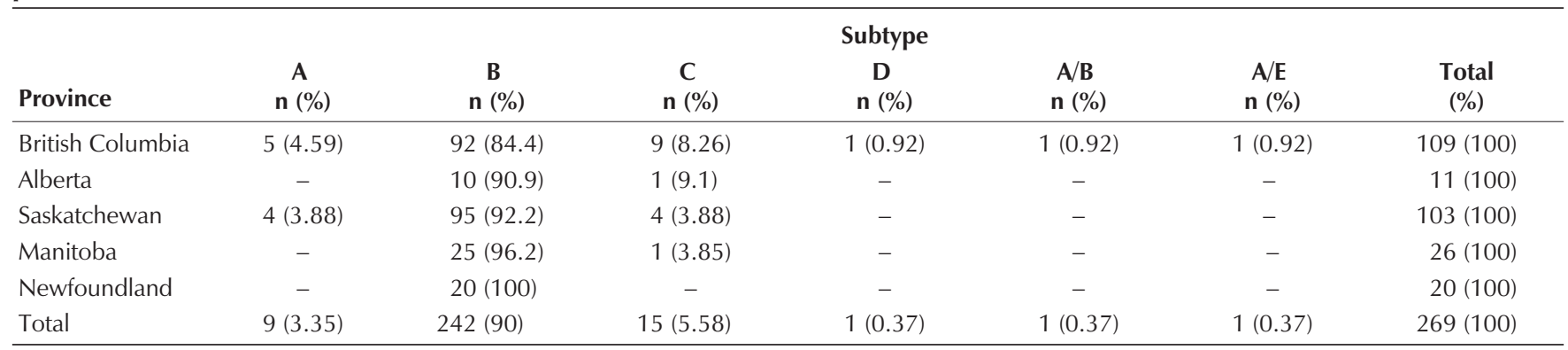


TABLE 4

Epidemiological information for non-B subtypes from British Columbia (Canadian HIV Strain and Drug Resistance Surveillance Program pilot site)

\begin{tabular}{lccc}
\hline Subtype & Sex & Risk factor & $\begin{array}{c}\text { Year of first } \\
\text { positive HIV test }\end{array}$ \\
\hline A & Male & MSM/IDU & 1996 \\
& Male & IDU & 1996 \\
& Male & MSM & 1997 \\
& Female & WSW & 1997 \\
C & Male & Heterosexual sex & 1998 \\
& Male & MSM/IDU & 1996 \\
& Female & Heterosexual sex & 1996 \\
& Male & Heterosexual sex & 1996 \\
& Male & IDU & 1996 \\
& Male & Heterosexual sex & 1996 \\
& Male & IDU & 1996 \\
& Male & MSM & 1996 \\
D & Male & MSM & 1996 \\
A/B & Male & Heterosexual sex & 1998 \\
A/E & Female & Heterosexual sex & 1998 \\
\hline & Male & MSM & 1996 \\
\hline
\end{tabular}

IDU Injection drug user; MSM Men who have sex with men; WSW Women who have sex with women

ducted using the statistical packages SPSS (SPSS Inc, Chicago, Illinois) and STATA (STATA Corporation, College Station, Texas).

Results: Of the 269 samples for which subtype analysis has been completed, 109 (40.5\%) were from British Columbia, 11 (4.1\%) were from Alberta, 103 (38.3\%) were from Saskatchewan, $26(9.7 \%)$ were from Manitoba and $20(7.4 \%)$ were from Newfoundland.

Two hundred and forty-two or $90 \%$ of the samples belonged to HIV-1 subtype B. Of the remaining 27 samples, nine $(3.3 \%)$ were of subtype A, 15 (5.6\%) were of subtype C, one (0.4\%) was of subtype D and one each $(0.8 \%)$ were of recombinant subtypes $\mathrm{A} / \mathrm{E}$ and $\mathrm{A} / \mathrm{B}$, respectively (Table 3 ).

The preliminary results reported above indicate geographic variation in the distribution of HIV-1 non-B subtypes. Whereas all 20 samples from Newfoundland were identified as subtype B, $15.6 \%$ of samples from British Columbia belonged to non-B subtypes. British Columbia also had the greatest genetic variation among the non-B HIV-1 subtypes (Table 3). Among the other pilot sites, the proportion of non-B subtypes was approximately $9.1 \%, 7.8 \%$, and $3.9 \%$, for Alberta, Saskatchewan and Manitoba, respectively. A more in-depth analysis of strains for each province is presented below. It should be noted that sample sizes in this interim analysis are small for some provinces and are not representative of Canada as a whole. It should also be noted that this report provides information only for those who were tested and diagnosed with HIV-1, and does not represent the total number of persons infected with HIV-1 in Canada.

HIV-1 subtypes for CHSDRSP samples from British Columbia: Stored serum specimen from individuals newly diagnosed with HIV in 1996, 1997, 1998, and up to March 15, 1999
TABLE 5

Epidemiological data of HIV-1 B versus non-B subtype for British Columbia (Canadian HIV Strain and Drug Resistance Surveillance Program pilot site)

\begin{tabular}{|c|c|c|c|}
\hline & $\begin{array}{c}\text { Subtype B } \\
n=92, \text { n (\%) }\end{array}$ & $\begin{array}{c}\text { Non-B subtype } \\
n=17, n(\%)\end{array}$ & $\mathbf{P}$ \\
\hline \multicolumn{4}{|l|}{ Sex } \\
\hline Female & $21(22.8)$ & $3(17.6)$ & 0.45 \\
\hline Male & $70(76.1)$ & $14(82.4)$ & \\
\hline Unknown & $1(1.09)$ & & \\
\hline \multicolumn{4}{|l|}{ Risk factor } \\
\hline $\mathrm{MSM}^{+}$ & $23(25)$ & $2(11.8)$ & 0.27 \\
\hline MSM/IDU & $2(2.17)$ & $2(11.8)$ & \\
\hline Heterosexual sex & $26(28.3)$ & $6(35.3)$ & \\
\hline IDU & $38(41.3)$ & $6(35.3)$ & \\
\hline Other $^{\ddagger}$ & $3(3.26)$ & $1(5.88)$ & \\
\hline $\begin{array}{l}\text { Mean age } \\
\text { (years } \pm \text { SD) }\end{array}$ & $40.7 \pm 9.9$ & $35.4 \pm 8.1$ & $0.04 *$ \\
\hline \multicolumn{4}{|c|}{ Year of first positive HIV test } \\
\hline 1996 & $21(22.8)$ & $11(64.7)$ & $0.003^{*}$ \\
\hline 1997 & $22(23.9)$ & $3(17.6)$ & \\
\hline 1998 & $25(27.2)$ & $3(17.6)$ & \\
\hline 1999 & $24(26.1)$ & $0(0)$ & \\
\hline \multicolumn{4}{|c|}{ Geographic area of residence } \\
\hline Vancouver city & $71(78.9)$ & $15(88.2)$ & 0.79 \\
\hline $\begin{array}{l}\text { Outside } \\
\text { metropolitian } \\
\text { Vancouver }\end{array}$ & $19(21.1)$ & $2(11.8)$ & \\
\hline \multicolumn{4}{|l|}{ Ethnicity } \\
\hline White & $58(63)$ & $8(47.1)$ & 0.32 \\
\hline Aboriginal & $20(21.7)$ & $4(23.5)$ & \\
\hline Other ${ }^{\S}$ & $14(15.2)$ & $5(29.4)$ & \\
\hline
\end{tabular}

*P indicates significant difference between HIV-1 B and non- $B$ subtypes using univariate analysis. ${ }^{+} \mathrm{MSM}$ includes men who have sex with men (MSM)/intravenous drug users (IDUs). Two MSM/IDUs each were infected with HIV-1 subtype $B$ and non-B, respectively. ${ }^{{ }^{\prime}}$ Other' risk categories include women who have sex with women (WSW), occupational exposure, male prostitute and infection with blood or blood products. ${ }^{\$}$ 'Other' ethnicities include Asian $(n=11)$, Black $(n=2)$ and Latin American $(n=3)$

were included in this pilot study. The samples represent between $4 \%$ to $6 \%$ of HIV-positive tests reported by British Columbia to the Division of HIV Surveillance, Bureau of HIV, STD and TB for each year (18), and were representative of exposure categories of those who were diagnosed with HIV for each year.

Ninety-two or $84.4 \%$ of the 109 samples from British Columbia that have been subtyped belonged to HIV-1 subtype B (Table 3$)$. Of the 17 non-B subtypes, five $(4.6 \%)$ belonged to subtype A, nine (8.3\%) were subtype C, one (0.9\%) was subtype $\mathrm{D}$, and one each were of recombinant subtypes $\mathrm{A} / \mathrm{B}$ and $\mathrm{A} / \mathrm{E}$. Additional epidemiological information from individuals infected with a non-B subtypes is shown in Table 4.

A comparison of epidemiological information of the HIV-1 subtype B and the non-B samples is shown in Table 5. Univariate analyses indicated no significant difference in sex or risk category between $B$ and the non-B subtypes. Among the sample population, those infected with a non-B subtype tended to be significantly younger (mean age 35.4 years) than those infected with the HIV-1 subtype B (mean age 40.7, P=0.04).

Univariate analysis also indicated a significant difference 
TABLE 6

Epidemiological profiles of subtype B samples from Alberta, Manitoba and Newfoundland (Canadian HIV Strain and Drug Resistance Surveillance Program pilot sites)

\begin{tabular}{|c|c|c|c|}
\hline & $\begin{array}{c}\text { Alberta } \\
n=10, n(\%)\end{array}$ & $\begin{array}{c}\text { Manitoba } \\
n=25, n(\%)\end{array}$ & $\begin{array}{c}\text { Newfoundland } \\
n=20, n(\%)\end{array}$ \\
\hline \multicolumn{4}{|l|}{ Sex } \\
\hline Female & $3(30 \%)$ & $5(20 \%)$ & $8(40 \%)$ \\
\hline Male & $7(70 \%)$ & $20(80 \%)$ & $12(60 \%)$ \\
\hline \multicolumn{4}{|l|}{ Risk factor } \\
\hline MSM & $3(30 \%)$ & $6(24 \%)$ & $4(20 \%)$ \\
\hline IDU & $4(40 \%)$ & $9(36 \%)$ & $1(5 \%)$ \\
\hline MSM/IDU & - & $1(4 \%)$ & - \\
\hline $\begin{array}{l}\text { Heterosexual } \\
\text { sex }\end{array}$ & $3(30 \%)$ & $9(36 \%)$ & $11(55 \%)$ \\
\hline Unknown & - & - & $4(20 \%)$ \\
\hline $\begin{array}{l}\text { Mean age } \\
\quad(\text { years } \pm S D)\end{array}$ & $34.5 \pm 12.9$ & $34.16 \pm 7.9$ & $32.7 \pm 6.07$ \\
\hline \multicolumn{4}{|l|}{ Ethnicity, n (\%) } \\
\hline White & $4(40 \%)$ & Not available & $20(100 \%)$ \\
\hline Aboriginal & $5(50 \%)$ & & \\
\hline $\begin{array}{l}\text { Latin } \\
\text { American }\end{array}$ & $1(10 \%)$ & & \\
\hline \multicolumn{4}{|c|}{ Year of first positive HIV test } \\
\hline 1985-1989 & - & $1(4 \%)$ & $3(15 \%)$ \\
\hline 1990-1994 & - & $3(12 \%)$ & $12(60 \%)$ \\
\hline 1995 & - & $2(8 \%)$ & $1(5 \%)$ \\
\hline 1996 & - & $2(8 \%)$ & - \\
\hline 1997 & - & - & $1(5 \%)$ \\
\hline 1998 & $9(90 \%)$ & $10(40 \%)$ & $1(5 \%)$ \\
\hline Unknown & $1(10 \%)$ & $7(28 \%)$ & $2(10 \%)$ \\
\hline
\end{tabular}

IDU Injection drug user; MSM Men who have sex with men

between $B$ and non-B subtypes with respect to year of first testing positive for $\mathrm{HIV}(\mathrm{P}=0.003)$, with the majority of non- $\mathrm{B}$ samples (11 of 17 ) in this interim analysis being diagnosed in 1996. There was no significant difference in subtype outcome among those diagnosed with HIV with respect to geographic residence or ethnicity. There were relatively more non-B subtypes found within metropolitan Vancouver and among ethnic groups other than White or Aboriginal persons, but neither association was statistically significant. Because of the low sample size in this interim analysis and the associated low statistical power, the results do not clearly differentiate between no true association and an association that simply was not detected by this analysis. It should be noted that two individuals in this group of samples identified themselves as Black, and both were infected with non-B subtypes, one with subtype A and the other with subtype D. Three of the 11 individuals who identified themselves as Asian were infected with non-B subtypes, two with subtype A and one with subtype C.

Logistic regression to determine the simultaneous effects among variables indicated that age and year of first testing positive for HIV were significantly associated with a non-B outcome ( $\mathrm{P}=0.009$ and $\mathrm{P}=0.0003$, respectively) in the sampled population. Compared with the B-subtype, the likelihood of a non-B subtype among those diagnosed with HIV was 1.11 times greater for every year decrease in age. The likelihood of infection with a non-B subtype in the sample population was 12.9 times higher among those diagnosed in 1996 compared with the three subsequent years combined. Ethnicity was of borderline significance in logistic regression; White and Aboriginal groups together were more likely to be diagnosed with B-subtype compared with other ethnic groups, but this association was not statistically significant at the $P=0.05$ level $(\mathrm{P}=0.071)$. Further analysis using a larger sample size is necessary to help elucidate this association.

HIV-1 subtypes for CHSDRSP samples from Alberta: Stored plasma specimens collected from individuals coming for viral load testing were included in this pilot study. The samples represent $9.3 \%$ of individuals reported as testing positive for HIV in Alberta during 1998 (18). Ten of the 11 samples (90.9\%) for which subtype analysis has been completed belonged to HIV-1 subtype B (Table 3). The epidemiological profile of these individuals is shown in Table 6 . The remaining sample was identified as HIV-1 subtype C. This sample was collected from a 30to 35-year-old female, infected through heterosexual transmission, who had moved to Canada from Africa and who first tested positive for HIV in 1998.

HIV-1 subtypes for CHSDRSP samples from Saskatchewan: Stored samples collected from individuals coming for viral load testing were included in this pilot study. Ninety-five of the 103 samples (92.2\%) from Saskatchewan were of HIV-1 subtype B (Table 3). Of the remaining eight samples, four belonged to subtype A (three women and one man) and four to subtype $\mathrm{C}$ (three men, one woman). Note that these 103 samples represent $24.8 \%$ of HIV diagnoses in Saskatchewan during 1985 to June 30, 1999 (18).

HIV-1 subtypes for CHSDRSP samples from Manitoba: Plasma samples collected from individuals coming for viral load testing, diagnosed with HIV-1 infection from a series of years before 1999, were selected for inclusion in this pilot study. These samples represent $0.85 \%, 7.1 \%, 8.3 \%$ and $38 \%$ of individuals reported as testing positive for HIV in 1985 to 1994, 1995, 1996 and 1998, respectively (18). Of the 26 samples on which subtype analysis was completed, 25 (96.2\%) belonged to HIV-1 subtype B (Table 3). The epidemiological profile of these individuals is shown in Table 6 . The remaining sample belonged to subtype $C$. It was collected from a male who was age 25 to 29 years, infected through heterosexual transmission and first tested positive in 1998.

HIV-1 subtypes for CHSDRSP samples from Newfoundland: Specimens collected from individuals coming for viral load testing with a wide variation in date of HIV diagnosis were included in this pilot study. These samples represent $9.6 \%$, $14.3 \%, 14.3 \%$ and $7.1 \%$ of individuals testing positive for HIV between 1985 and 1994, 1995, 1997 and 1998, respectively (18). All 20 individuals in the sample population from Newfoundland were infected with HIV-1 subtype B (Table 3). The epidemiological profile of these individuals is shown in Table 6 .

\section{DISCUSSION}

At the end of 1996, there were approximately 40,000 Canadians living with HIV infection (19). The number of incident HIV infections in Canada for 1996 was approximately 4200, 
higher than the average of 2500 to 3000/year from 1989 to 1994 (19). An analysis of the distribution of incident infections by exposure category illustrates a changing face of the HIV epidemic in Canada, where now nearly one-half of all new infections can be attributed to injection drug use (19). Women are increasingly affected as are minority populations.

In addition to the aforementioned changes in the populations infected with HIV, the present results confirm that a significant proportion of non-B HIV-1 subtypes has been introduced into Canada and suggest that there is likely geographic variation in the prevalence of these subtypes. From the sample population analyzed for this interim report, $10 \%$ were infected with a non-B subtype, of which $3.3 \%$ were of subtype A, $5.6 \%$ were of subtype $\mathrm{C}$ and $0.4 \%$ were of subtype $\mathrm{D}$.

It should be emphasized that the sample population represents those diagnosed with HIV from a variety of years and is not reflective of the total population infected with HIV. Furthermore, these results may not be representative of the respective provinces, with respect to HIV strains that are currently circulating. The percentage of HIV test positive reports represented in the sample population varies between the respective provinces. For example, up to $38 \%$ of all positive HIV tests reported for 1998 from Manitoba are represented in this report, whereas only $9.3 \%, 7.1 \%$, and $5.8 \%$ of positive HIV tests reported for 1998 are represented from Alberta, Newfoundland and British Columbia, respectively.

Despite these limitations, our findings are similar to those documented for other countries where subtype B used to predominate. In Sweden, until 1984, the HIV epidemic was almost entirely caused by subtype B; however, by 1993, non-B subtypes A and C, accounted for $30 \%$ of all new infections (20). In a pilot study conducted in Germany, eight of 24 recent HIV-1 infections among German nationals (33\%) were because of non-B subtypes including subtypes A, C, and E (21). In England, subtypes A, C, D, and A/E have been identified (22), and in Belgium, subtypes A, C, D, F, G, and H were reported in 1996 (23). In the United States, subtype D was identified as early as 1993 (24). Sentinel site surveillance of HIV in the United States has also indicated the introduction of other non-B subtypes into the country. A non-blinded study in 1992 to 1994 at the Bronx-Lebanon Hospital Center (Bronx, New York) found HIV-1 subtype A in two of $43(5 \%)$ patients (25). A follow-up study found that among $91 \mathrm{HIV}-1$ infected patients, one (1.1\%) was infected subtype A and two (2.2\%) with subtype F (26). In 1998, the Centers for Disease Control and Prevention, Atlanta, Georgia (CDC) initiated a program for the sentinel surveillance of strain and drug resistance nationwide. Results from this study suggest that $1.6 \%$ of newly diagnosed (within the past year) HIV-infected persons, who are at least 18 years of age and have no history of AIDS-defining illnesses according to the 1993 CDC AIDS case definition, are infected with a non-B subtype. Specifically, subtype A has been identified in five persons in a sample population of 321 (27).

While the CHSDRSP reference services and the pilot study have identified HIV-1 non-B subtypes in most provinces, the present results suggest that the proportion of non-B subtypes varies between geographic regions. The CHSDRSP pilot study identified no non-B subtypes from Newfoundland, whereas up to $15.6 \%$ of the samples from British Columbia belonged to this group. However, a specimen from an African-born person in Newfoundland was identified as subtype A by the CHSDRSP reference services. Also, the only sample identified as subtype $D$ from the CHSDRSP pilot study was collected from an individual of African origin. Further phylogenetic analysis is required to confirm whether the relatively high proportion of non-B subtypes in British Columbia may be related to a cluster of infections several years ago, as suggested by the majority of non-B subtypes being found in 1996. Cluster investigations similar to the study conducted by Montpetit et al (7) in Newfoundland would also help clarify this matter.

A higher prevalence of non-B subtypes may be directly related to the presence of mobile populations and immigration from countries where divergent HIV subtypes are found. More data collection and analysis are required to confirm this hypothesis, but the impacts of travel and migration on blurring geographic distinctions of subtype distribution are well documented. The deployment of army personnel, for example, has been associated with the introduction of non-B subtypes into the United States (28), Uruguay (29) and France (30). Non-B subtypes have also been identified among immigrant populations originating from HIV-endemic countries living in the United States (27,31), England (22), Sweden (32), Netherlands (33), France (34) and Israel (35).

The introduction of variant HIV subtypes into Canada will invariably challenge existing diagnostic tests and/or interpretation algorithms. Depending on future findings related to the transmissibility, pathogenicity and treatment implications of various subtypes, it may also play a role in changing the nature of the HIV epidemic in Canada. It is, therefore, imperative to implement a systematic collection and analysis of data related to strain surveillance across Canada.

\section{FUTURE DIRECTIONS}

Although the use of highly active antiretroviral therapy (HAART) has led to a reduction in HIV-1 related morbidity and mortality in the developed world, HAART has created a unique set of challenges - the development of resistance to antiretroviral drugs that greatly affect the treatment of HIV-1 infection. Much more remains to be learned about the transmission of drug-resistant strains and the implications for the clinical management of individuals infected with antiretroviral resistant HIV.

One of the central goals of CHSDRSP is to monitor drug-resistant strains of HIV, in particular to assess the extent of transmission of drug-resistant HIV-1 variants to drug-naïve individuals. This information may be used to guide clinical management decisions and to develop guidelines for initial therapy at the population level. It may also help enhance coverage for the treatment of newly diagnosed HIV-positive cases in Canada. The epidemiological component of resistance surveillance consists of an existing HIV surveillance database plus additional information collected to help interpret the laboratory information. The laboratory component involves the collection of serum from all new HIV diagnoses for sequencing of polymerase gene pol PCR products to determine 
mutations associated with resistance to individual or groups of antiretroviral drugs.

We expect that preliminary drug resistance results will be available within a few months, and the CHSDRSP pilot will be expanded to include all provinces and territories by the end of 2000.

ACKNOWLEDGEMENTS: The National Laboratory for HIV Genetics, Bureau of HIV/AIDS, STD and TB was responsible for the subtype results presented under CHSDRSP Initiatives. The authors acknowledge and thank the following individuals and organizations involved in the collection and submission of serum and plasma specimens, and epidemiological data: British Columbia (M Rekart, D Patrick and D Cook, British Columbia Centre for Disease Control, Vancouver), Alberta (B Larke, S Houston and A Chow, Alberta Health and Wellness, Edmonton); Saskatchewan (E Young, G Horsman, E Chan and F Sidaway, Saskatchewan Health, Regina); Manitoba (P Matusko and M Dawood, Manitoba Health, Winnipeg); Newfoundland (S Ratnam, Newfoundland Public Health Laboratory, St John's; F Stratton, Department of Health and Community Services, St John's). Thanks also to Drs M Rekart, B Larke, S Houston, P Matusko and S Ratnam for their helpful comments on this report. Special thanks to Drs Mike Montpetit and Paul Sandstrom for critically reviewing this report.

\section{REFERENCES}

1. Mansky LM, Temin HM. Lower in vivo mutation rate of HIV type 1 than that predicted from the fidelity of purified reverse transcriptase. J Virol 1995;69:5087-94.

2. Subbarao S, Schochetman G. Genetic variability of HIV-1. AIDS 1996;10(Suppl A):S13-S23.

3. Myers G, Korber B, Foley B, et al, eds. Human Retroviruses and AIDS. A Compilation and Analysis of Nucleic Acid and Amino Acid Sequences. Los Alamos: Theoretical Biology and Biophysics, 1996.

4. Gurtler L, Hauser PH, Eberle J, et al. A new subtype of HIV type 1 (MVP-5180) from Cameroon. J Virol 1994;68:1581-5.

5. Simon F, Mauclere P, Roques P, et al. Identification of a new human immunodeficiency virus type 1 distinct from group $M$ and group O. Nat Med 1998;4:1032-7.

6. Bernier L, Lamothe F, Bruneau J, et al. Analysis of HIV-1 sequence diversity in infected IDUs and MSM from Montreal. Eighth Annual Canadian Conference on HIV/AIDS Research, Vancouver, May 1-4, 1999. [Abst A104]

7. Montpetit ML, Ratnam S, Campbell C, Gleeson T, Donovan C, Tollefson J. Molecular epidemiological analysis of human immunodeficiency virus type 1 in Newfoundland, Canada. AIDS Res Hum Retroviruses 1998;14:1205-9.

8. Major C, Calzavara L, Cassol S, et al. Strain Surveillance and Genotyping, POLARIS, Ontario. Proceedings from the Joint HIV/AIDS and STD Meeting, Bureau of HIV/AIDS, STD and TB, Laboratory Centre for Disease Control, Health Canada. Calgary, November17-20, 1999. (In press)

9. Harrigan PR, Dong W, Alexander CS, et al. HIV transmission networks in seroconverting injection drug users. Eighth Annual Canadian Conference on HIV/AIDS Research. Vancouver, May 1-4, 1999. [Abst A103]

10. Montpetit M. HIV-1 subtype A in Canada. AIDS Res Hum Retroviruses 1995;11:1421-2.

11. Alexander C, Dong W, Chan K, et al. HIV non-B subtypes in a large North American cohort: prevalence and response to antiretroviral therapy. Seventh Conference on Retroviruses and Opportunistic Infections. San Francisco, January 31-February 3, 2000. [Abst 174]

12. Harrigan PR. HIV strain methods and results from the BC Centre for Excellence in HIV/AIDS. Proceedings from the Joint HIV/AIDS and STD Meeting, Bureau of HIV/AIDS, STD and TB, Laboratory Centre for Disease Control, Health Canada. Calgary, November 17-20, 1999. (In press)

13. Kunanusont C, Foy HM, Kreiss JK, et al. HIV-1 subtypes and male-to-female transmission in Thailand. Lancet 1995;345:1078-83.

14. Hu DJ, Buve A, Baggs J, van der Groen G, Dondero TJ. What role does HIV-1 subtype play in transmission and pathogenesis? An epidemiological perspective. AIDS 1999;13:873-81.

15. Alexander C, Dong W, Chan K, et al. HIV non-B subtypes in a large North American cohort: prevalence and response to antiretroviral therapy. Seventh Conference on Retroviruses and Opportunistic Infections. San Francisco, January 31February 3, 2000. [Abst 174].

16. Loussert-Ajaka I, Ly TB, Chaix ML, et al. HIV-1/HIV-2 seronegativity in HIV-1 subtype 0 infected patients. Lancet 1994;343:1393-4

17. Boom R, Sol CJA, van der Noorda J, et al. Rapid and simple method for purification of nucleic acids. J Clin Microbiol 1990;35:273-86.

18. HIV and AIDS in Canada. Surveillance Report to June 1999 Bureau of HIV/AIDS, STD and TB, Laboratory Centre for Disease Control. Ottawa: Health Canada, November 1999.

19. HIV Prevalence and Incidence in Canada: 40,100 living with HIV infection and 4,200 new infections per year. HIV/AIDS and STD EpiUpdate. Bureau of HIV/AIDS, STD and TB, Laboratory Centre for Disease Control. Ottawa: Health Canada, May 1999.

20. Sonnerborg A, Durdevic S, Giesecke J, Sallberg M. Dynamics of the HIV-1 subtype distribution in the Swedish HIV-1 epidemic during the period 1980 to 1993 . AIDS Res Hum Retroviruses 1997; 13:343-5.

21. Dietrich U, Ruppach H, Gehring S, et al. Large proportion of non-B HIV-1 subtypes and presence of zidovudine resistance mutations among German seroconverters. AIDS 1997;11:1532-3.

22. Arnold C, Barlow KL, Parry JV, Clewley JP. At least five HIV-1 sequence subtypes (A,B,C,D,A/E) occur in England. AIDS Res Hum Retroviruses 1995;11:427-9.

23. Fransen K, Buve A, Nkengasong JN, van der Groen G. Longstanding presence in Belgians of multiple non-B HIV-1 subtypes. Lancet 1996;347:1403.

24. Gao F, Yue L, Hill SC, et al. HIV-1 sequence subtype D in the United States. AIDS Res Hum Retroviruses 1993;10:625-7.

25. Irwin KL, Pau CP, Lupo D, et al. Presence of human immunodeficiency virus (HIV) type 1 subtype A infection in a New York community with high HIV prevalence: A sentinel site for monitoring HIV genetic diversity in North America. Centers for Disease Control and Prevention-Bronx Lebanon HIV Serosurvey Team. J Infect Dis 1997;176:1629-33.

26. Weidle PJ, Ganea CE, Pienniazek D, et al. Prevalence of HIV-1 group $\mathrm{M}$, non-B subtypes in a Bronx, New York community: A sentinel site for monitoring HIV genetic diversity in the United States. 12th World AIDS Conference. Geneva, June 28-July 3, 1998. [Abst 13225]

27. Zaidi I, Weinstock H, Kalish ML et al. Surveillance for HIV-1 subtypes in the United States, 1998-present. 7 th Conference on Retroviruses and Opportunistic Infections. January 31February 3, 2000. [Abst 215]

28. Brodine SK, Mascola JR, Weiss PJ, et al. Detection of diverse HIV-1 genetic subtypes in the USA. Lancet 1995;346:1198-9.

29. Artenstein AW, Copola J, Brown AE, et al. Multiple introductions of HIV-1 subtype E into the western hemisphere. Lancet 1995;346:1197-8.

30. Lasky M, Perret JL, Peeters M, et al. Presence of multiple non-B subtypes and divergent subtype B strains of HIV-1 in individuals infected after overseas deployment. AIDS 1997;11:43-51.

31. Sullivan P, Do AN, Lal R, et al. Surveillance for Group O HIV-1 infections among African-born persons in the United States 1995-1997. 12th National AIDS Conference. Geneva. June 28-July 3, 1998. [Abst 13103]

32. Alaeus A, Leitner T, Lidman K, Albert J. Most HIV-1 genetic subtypes have entered Sweden. AIDS 1997;11:199-202.

33. Lukashov VV, Kuiken CL, Boer K, Goudsmit J. HIV type 1 subtypes in The Netherlands circulating among women originating from AIDS-endemic regions. AIDS Res Hum Retroviruses 1996;12:951-3.

34. Simon F, Loussert-Ajaka, I, Damond F, et al. HIV type 1 diversity in northern Paris, France. AIDS Res Hum Retroviruses 1996;12:1427-33.

35. Gehring S, Maayan S, Ruppach H, et al. Molecular epidemiology of HIV in Israel. J Acquir Immune Defic Syndr Hum Retrovirol 1997;15:296-303. 


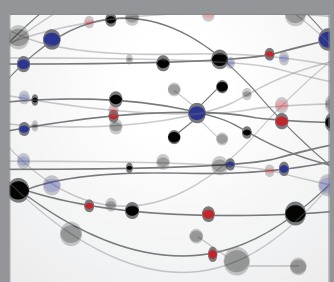

The Scientific World Journal
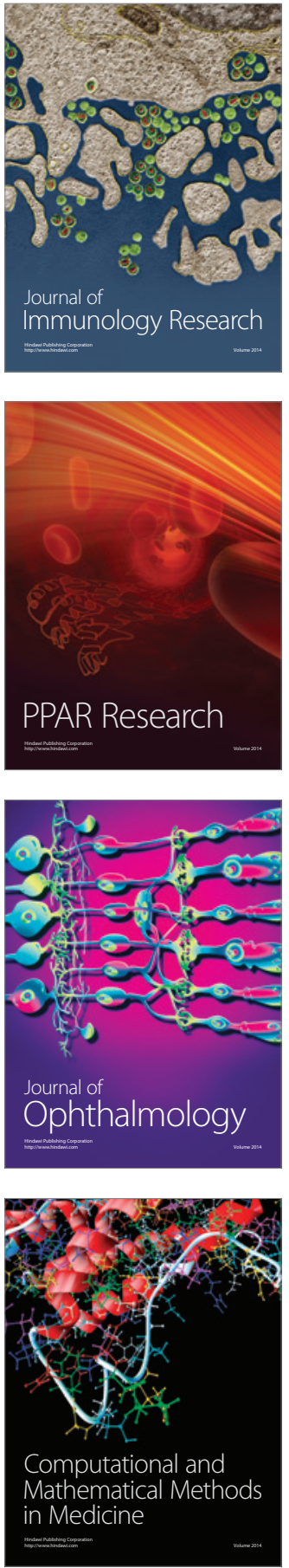

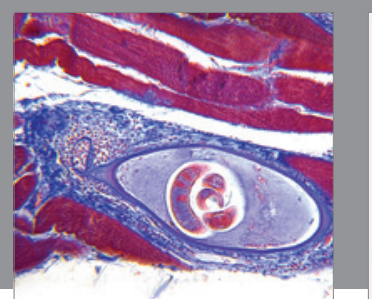

Gastroenterology Research and Practice

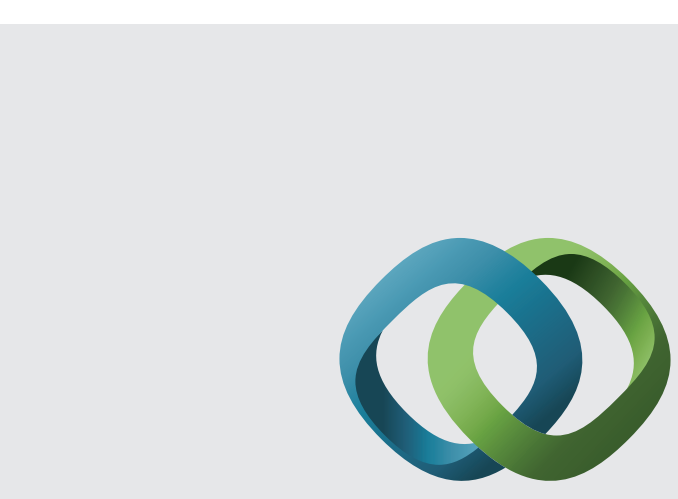

\section{Hindawi}

Submit your manuscripts at

http://www.hindawi.com
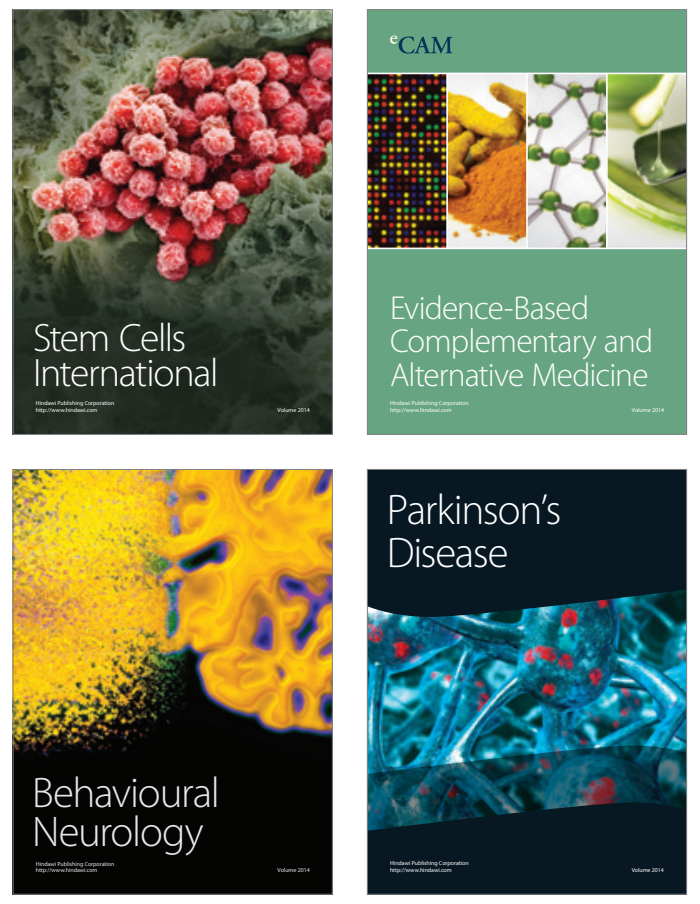
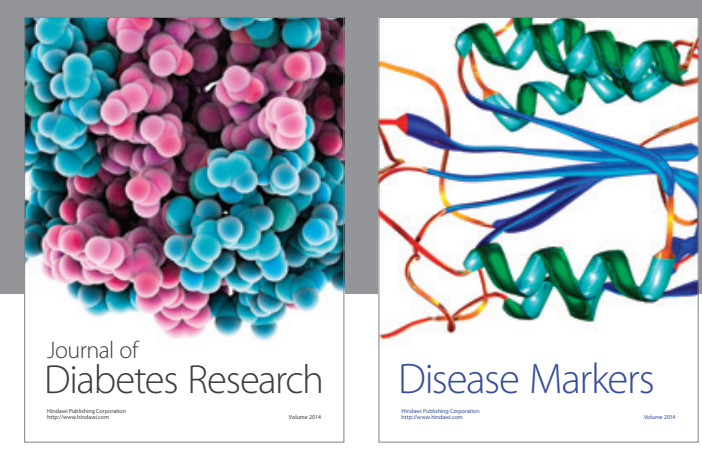

Disease Markers
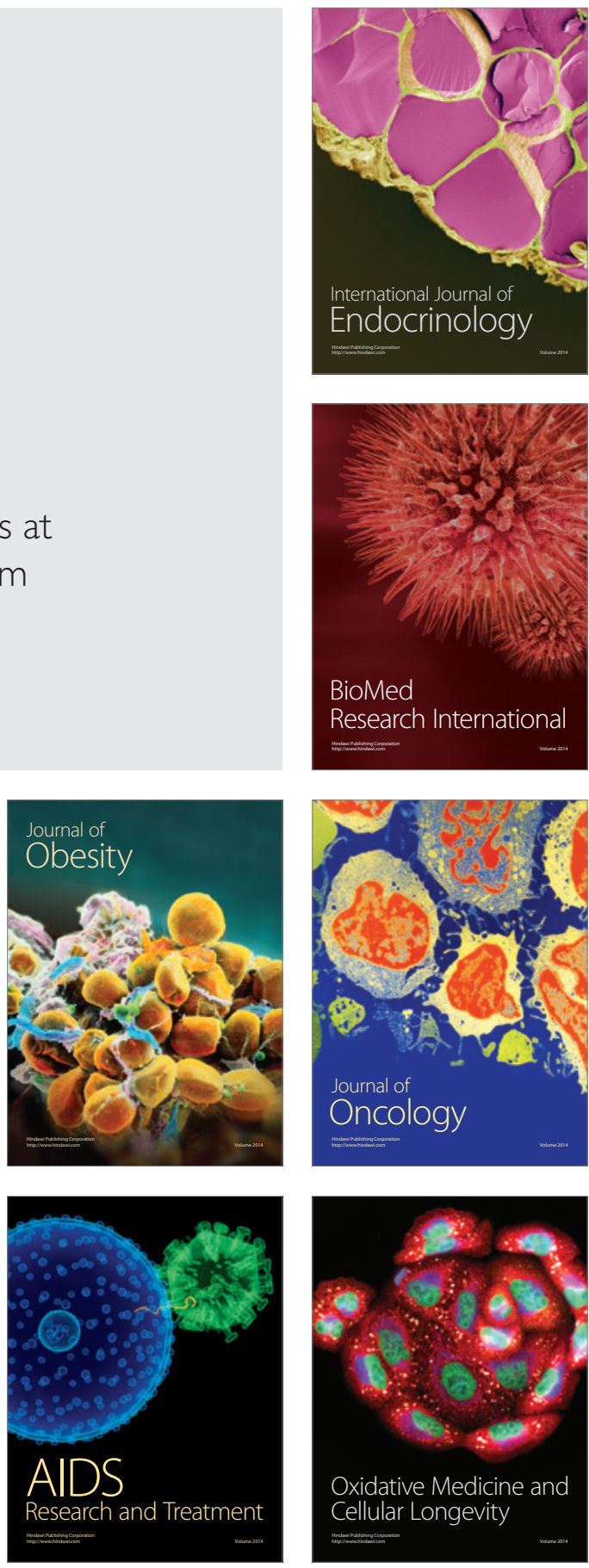